\title{
Artigo de Reisão
}

\section{FIBROSE SISTÊMICA NEFROGÊNICA: UMA COMPLICAÇÃO GRAVE DO USO DO GADOLÍNIO EM PACIENTES COM INSUFICIÊNCIA RENAL}

\author{
Juliano Sacramento Mundim*1, Sabrina de Castro Lorena², Hugo Abensur ${ }^{3}$, Rosilene Motta Elias ${ }^{4}$, Rosa Maria Affonso Moysés ${ }^{4}$, Manuel Carlos Martins de \\ CASTRO ${ }^{4}$, JoÃo EGídIO ROMÄO JÚNIOR ${ }^{5}$ \\ Trabalho elaborado no Serviço de Nefrologia do Hospital das Clínicas da Faculdade de Medicina da Universidade de São Paulo, São Paulo, SP
}

*Correspondência:

Rua Barata Ribeiro,

número 360 , apto 1007

CEP: $01308-000$

Bela Vista - São Paulo - SP

\begin{abstract}
RESUMO
Fibrose sistêmica nefrogênica (FSN), também conhecida como dermopatia fibrosante nefrogênica (DFN), é uma condição que ocorre apenas em pacientes com disfunção renal. Além das lesões cutâneas, esta síndrome inclui fibrose de músculo esquelético, articulações, fígado, pulmão e coração e pode ser fatal. Esta doença foi primeiramente descrita em 1997 e vários estudos descrevem a relação etiológica da FSN com a exposição a agentes de contraste contendo gadolínio, usado em exames de ressonância magnética. Esta revisão tem como objetivo alertar médicos clínicos e nefrologistas sobre essa nova patologia que acomete pacientes com alteração da função renal, demonstrando aspectos demográficos e epidemiológicos, apresentação clínica, diagnóstico e prognóstico além das opções de prevenção e terapêuticas atuais. Concluímos que todo paciente apresentando creatinina sérica elevada deve ter sua função renal (clearance de creatinina) estimada, visando a segurança na realização da ressonância magnética.
\end{abstract}

KeY WORDS: Fibrose sistêmica nefrogênica. Dermopatia fibrosante sistêmica. Gadolínio. Fibrose. Insuficiência renal.

\section{INTRODUÇÃO}

Reconhecida primeiramente em $1997^{1}$, a dermopatia fibrosante nefrogênica (DFN) foi identificada em 15 pacientes portadores de insuficiência renal crônica em programa dialítico, que desenvolveram espessamento e endurecimento da pele com forte hiperpigmentação, pápulas e nódulos subcutâneos nas extremidades. Desde a primeira publicação, a DFN tem sido vista exclusivamente em pacientes com insuficiência renal e afeta principalmente a pele e o tecido subcutâneo.

Casos recentes descrevem o acometimento de outros órgãos, mostrando que a DFN é uma doença sistêmica ${ }^{2,3,4}$; assim, a denominação de fibrose sistêmica nefrogênica (FSN) foi considerada para melhor defini-la $a^{5}$. Outras terminologias como doença escleromixedema-like da doença renal, doença escleromixedema-like da hemodiálise, escleromixedema-like fibromucinosa, fibrose sistêmica associada à diálise, podem ser encontradas na literatura em associação com esta entidade ${ }^{1,3,6,7}$. Discutiremos nesta revisão as características desta doença, alertando aos clínicos e nefrologistas a sua gravidade e mostrando as atuais propostas terapêuticas.

\section{Epidemiologia}

A FSN ocorre exclusivamente em pacientes com insuficiência renal, com clearance estimado de creatinina $<30 \mathrm{ml} / \mathrm{min}$ (clearance de creatinina estimado $=\{[(140-$ Idade em anos $) \mathrm{x}$ Peso em kg] / [72 x creatinina, em mg/dL] x 0,85 se mulher $\}$ ) envolvendo principalmente pacientes em hemodiálise, mas também em diálise peritoneal, receptores de transplante renal (tipicamente com redução da função renal), pacientes com doença renal crônica avançada e com insuficiência renal aguda sem necessidade de diálise ${ }^{8}$.

Até dezembro de 2006, aproximadamente 215 casos de FSN foram descritos para o Registro Internacional de FSN da Universidade de Yale, nos Estados Unidos ${ }^{9}$. A maioria dos

1. Residência de Clínica Médica no Hospital Municipal de São J osé dos Campos -SP - Residência Médica de Nefrologia na Faculdade de Medicina da Universidade de São Paulo, São Paulo, SP

2. Residência Médica de Nefrologia na Faculdade de Medicina da Universidade de São Paulo - Médica Preceptora da Residência Médica de Nefrologia na Faculdade de Medicina da Universidade de São Paulo, São Paulo, SP

3. Professor Livre-docente da Nefrologia da Faculdade de Medicina da Universidade de São Paulo - Médico Assistente da Unidade de Hemodiálise do Hospital das Clínicas da faculdade de Medicina da Universidade de São Paulo, São Paulo, SP

4. Doutora em Nefrologia pela Faculdade de Medicina da Universidade de São Paulo - Médica assistente da unidade de hemodiálise do Hospital das Clínicas da Faculdade de Medicina da Universidade de São Paulo, São Paulo, SP.

5. Professor Livre-docente da Nefrologia da Faculdade de Medicina da Universidade de São Paulo - Chefe da Unidade de Hemodiálise do Hospital das Clínicas da Faculdade de Medicina da Universidade de São Paulo, São Paulo, SP 
pacientes eram adultos, mas crianças podem ser afetadas ${ }^{10}$. Não há predileção pelo gênero, raça ou idade, etiologia ou duração da insuficiência renal ${ }^{11-14}$. Entretanto, pacientes submetidos à diálise peritoneal, comparados à hemodiálise, parecem apresentar maior risco ${ }^{15}$.

A relação entre o tempo do início da diálise e o diagnóstico de FSN variou de 2 meses a 15 anos, segundo uma série de 10 $\operatorname{casos}^{16}$. Já outras séries relataram que o tempo médio da exposição ao gadolínio até o aparecimento de evidências clínicas de FSN foi de 25 dias (variando de 2 a 75 dias) ${ }^{17,18}$.

\section{Etiologia}

A etiologia da FSN é pouco conhecida e esta nova doença resulta provavelmente da exposição dos pacientes com insuficiência renal a uma nova medicação, agente infeccioso ou toxina.

Evidências epidemiológicas atuais apontam para a possibilidade de esta doença estar envolvida com o agente de contraste contendo gadolínio, usado para a realização de ressonância nuclear magnética ${ }^{19,} 20$.

\section{Características e farmacocinética do gadolínio}

O gadolínio é um raro elemento metálico lantanóide com propriedades ferromagnéticas. Por ser um metal, deve estar na forma iônica $\left(\mathrm{Gd}^{3}\right)$ para dissolver na água e funcionar como meio de contraste. Entretanto, o $\mathrm{Gd}^{3}$ é muito tóxico e pode precipitar em vários tecidos (fígado, nódulos linfáticos e osso), bloquear o transporte de cálcio nas células musculares e nervosas diminuindo a transmissão neuromuscular, e também interferir com enzimas intracelulares e membrana celular por um processo de transmetalação. Por isso, o Gd é administrado em associação com moléculas orgânicas maiores (quelantes), formando complexo mais estável, evitando os efeitos tóxicos do $\mathrm{Gd}^{3}$ e dificultando a transmetalação ${ }^{21}$.

A transmetalação é facilitada pelo Zinco, Cobre, Ferro, Cálcio ou ácidos endógenos, que desestabilizam tais complexos, liberando $0 \mathrm{Gd}^{3}$. Este é pouco solúvel, e, após extravasamento anormal vascular (trauma, edema crônico, disfunção endotelial), pode formar precipitados de sais de fosfato, sendo depositados no interstício, e, então, fagocitados por macrófagos que produzem citocinas prófibróticas ${ }^{17}$.

Todos os meios de contraste contendo gadolínio possuem quelantes na sua composição, existindo duas categorias estruturais: as macrocíclicas, que possuem o $\mathrm{Gd}^{3}$ localizado no interior da molécula, dificultando sua liberação e conferindo maior proteção, e as lineares, mais instáveis. E quanto à carga, podem ser iônicas e não-iônicas ${ }^{22}$. As formulações de gadolínio aprovados pelo FDA (Food and Drug Administration) estão na Tabela 1.

A dose usual para estudos não vasculares é de $0,1 \mathrm{mmol} / \mathrm{kg}$, sendo mais elevada para estudos de angioressonância.

Em indivíduos com função renal normal (tabela 2), 0 gadolínio se equilibra rapidamente entre o plasma e 0 interstício, com meia-vida de 2 horas e é eliminado através da filtração glomerular, sem contribuição da secreção tubular, com clearance renal variando de 1,1 a 1,6 ml/kg/min. Mais de $95 \%$

\begin{tabular}{lccc}
\hline Tabela 1 - Agentes de contraste contendo gadolínio liberados pelo FDA \\
\hline Formulação do Gadolínio & $\begin{array}{c}\text { Osmolaridade } \\
\text { mOsm/kg) }\end{array}$ & Carga & $\begin{array}{r}\text { Estrutura } \\
\text { molecular }\end{array}$ \\
\hline Gadopentetate dimeglumine & 1960 & iônico & linear \\
Gadodiamide & 650 & não iônico & linear \\
Gadoteridol & 630 & não iônico & cíclico \\
Gadobenate dimeglumine & 1970 & iônico & cíclico \\
Gadoversetamide & 1110 & não iônico & linear \\
\hline
\end{tabular}

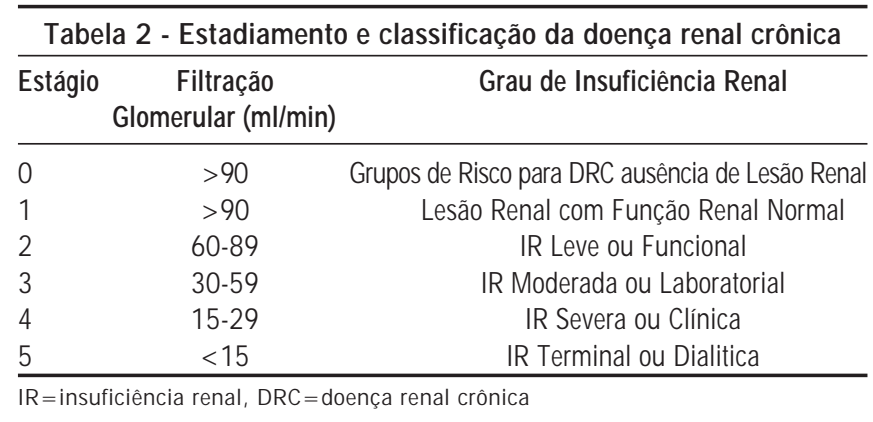

da dose injetada é eliminada em $24 \mathrm{hs}$ e menos de $3 \%$ eliminada nas fezes.

Em pacientes com insuficiência renal avançada (estágio 5) a farmacocinética dos complexos com gadolínio está alterada. Devido ao relativo baixo peso molecular (500 Da), pequeno volume de distribuição $(0,28 \mathrm{l} / \mathrm{kg})$ e pouca ligação protéica, estes são facilmente removíveis com hemodiálise, mas não pela diálise peritoneal ${ }^{21}$.

Em um estudo, a meia-vida do gadolínio em paciente com doença renal crônica foi de 34,3 horas e diminuiu para 2,6 horas nos que receberam hemodiálise, enquanto que nos pacientes mantidos em diálise peritoneal permaneceu por 52,7 horas $^{23}$.

\section{Fisiopatologia}

A sequência dos eventos fisiopatológicos desta grave doença fibrótica ainda não está clara; entretanto, encontrase na imunohistoquímica um infiltrado cutâneo inicial com células dendríticas , CD68 / fator XIIIa que poderia representar uma possível resposta do hospedeiro ao estímulo nocivo. Além disso, estudos com hibridização in situ e imunohistoquímica mostraram o aumento de um marcador de expressão do TGF $\beta 1$ no RNAm, distribuído difusamente na pele, fáscia e músculos afetados. Assim, há a hipótese de que o TGF $\beta 1$ estaria intimamente envolvido no processo fisiopatológico da doença ${ }^{16}$.

A eritropoetina recombinante humana (EPO) foi implicada com FSN devido às suas propriedades fibrogênicas ${ }^{24}$, estimulando a medula óssea e um grande número de fibroblastos CD34 derivados da medula óssea a infiltrar na derme dos pacientes com FSN. A EPO foi colocada como o possível agente etiológico da FSN num estudo comparativo com 22 pacientes em hemodiálise com biópsia compatível com FSN e 50 pacien- 
tes controles em hemodiálise ${ }^{25}$, em que os pacientes com FSN receberam dose média de EPO significativamente maiores que o grupo controle (427 U/kg versus $198 \mathrm{U} / \mathrm{kg}$ por semana). A FSN melhorou em alguns pacientes que pararam ou diminuíram a dose de EPO. Entretanto, não está claro se este efeito ocorreu pela redução da terapia ou recuperação da função renal. Há necessidade de mais estudos para comprovar se há alguma associação entre EPO e FSN.

Como outra teoria, o efeito estimulante para a FSN pode ser o depósito tecidual de uma toxina, como o gadolínio. $0 \mathrm{Gd}^{3}$ é pouco solúvel, altamente tóxico e pode se precipitar com outros ânions, geralmente aumentados na insuficiência renal, dissociando-se dos quelantes, ${ }^{15,21,24-27}$ levando ao processo já descrito anteriormente.

\section{Achados Clínicos e laboratoriaIS \\ Manifestações cutâneas}

A lesão primária aparece na parte distal das extremidades na maioria dos pacientes, como nas mãos, levando à esclerodactilia, seguida da parte proximal das extremidades e tronco. Face e pescoço são poupados ${ }^{1,2,16}$.

Em muitos casos, edema das extremidades afetadas tem sido descrito antes do aparecimento das lesões características. Quando este edema está presente, ele gradualmente desaparece, deixando placas rígidas e eritematosas, que progridem para espessamento, com aparência de "casca de laranja", podendo acometer áreas em faixas, semelhantes às encontradas na esclerodermia (figura 1).

Progressivamente, este espessamento envolve os tecidos peri-articulares, provocando limitações da motilidade e contraturas em flexão (Figura 1). A cronologia do acometimento visceral não está clara, mas é mais comum em pacientes com acometimento cutâneo extenso ${ }^{16}$.

A realização da biópsia de pele é mandatória sendo necessária uma biópsia profunda, pois as lesões fibróticas podem se estender além do tecido subcutâneo. A microscopia varia de acordo com a gravidade da doença, encontrando desde proliferação cutânea sutil de fibrócitos nas lesões iniciais, até espessamento da derme com proliferação difusa de fibrócitos com longos processos dendríticos nos casos avançados.

O prognóstico depende da extensão, gravidade e rapidez do envolvimento cutâneo, e a gravidade do envolvimento sistêmico ${ }^{1,15,16,21,25,26}$.

\section{Manifestações sistêmicas}

A primeira descrição do envolvimento sistêmico foi a de um paciente que na autópsia revelou fibrose e calcificação do diafragma e músculo psoas e testículos ${ }^{2}$.

0 acometimento de tecidos periarticulares e tendões pode levar a diminuição dos movimentos e contraturas em flexão, sem evidências clínicas de sinovite ou artrite ${ }^{16}$.

Os músculos podem ser acometidos sem que haja manifestação clínica de fraqueza muscular. Quando há acometimento nervoso periférico, se observa a presença de neuropatia motora e sensitiva sem que se saiba ao certo se está relacionado a FSN ou a doença renal em estágio final.

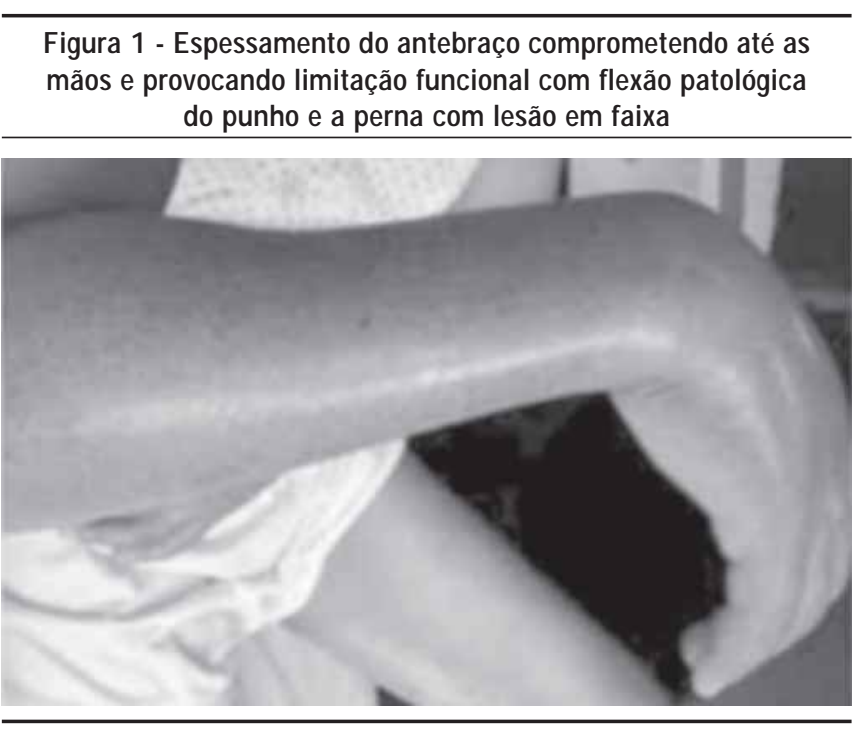

Quanto ao envolvimento cardíaco, a análise de um paciente por autópsia mostrou alterações fibróticas no miocárdio ${ }^{16}$.

Em alguns pacientes, há alteração do teste de função pulmonar. Outros acometimentos sistêmicos incluem trombose de fístula arteriovenosa para hemodiálise, oclusão vascular periférica, ataque isquêmico transitório e infartos cerebrais múltiplos ${ }^{16}$.

\section{Achados laboratoriais}

Não há achados laboratoriais específicos associados a FSN . Entretanto, os pacientes acometidos possuem alterações séricas consistentes com inflamação crônica, incluindo elevação da proteína C reativa, ferritina sérica, velocidade de hemossedimentação, e uma redução na albumina sérica. ${ }^{16}$

0 teste de função pulmonar pode revelar uma redução na capacidade pulmonar total e volume e capacidade de difusão nos casos de acometimento extra-renal ${ }^{16}$. A biópsia muscular deve ser obtida para determinar a extensão do envolvimento sistêmico.

\section{Diagnóstico diferencial}

Os principais diagnósticos diferenciais com a FSN são patologias que acometem a pele, levando ao espessamento e endurecimento da epiderme e derme. Entretanto, a presença de insuficiência renal é fortemente sugestiva de FSN. As principais patologias são:

Esclerodermia - é uma patologia sistêmica, associada a anticorpos circulantes (anti-centrômero, Anti-SCL 70) e ao fenômeno de Raynaud, e a ausência dessas características falam contra esta patologia.

Escleromixedema - trata-se de uma patologia sistêmica rara e com aparecimento de placas ou pápulas principalmente na região do pescoço e cabeça, regiões geralmente poupadas na FSN, associadas à deposição de glicosaminoglicanos e proliferação de fibroblastos e frequentemente com pico monoclonal ${ }^{1,6}$. 
Fasceíte eosinofílica - a presença de eosinofilia e eosinófilos nos tecidos envolvidos faz parte do diagnóstico desta doença ${ }^{28,29}$.

Calcifilaxia - desordem comum em pacientes com insuficiência renal crônica devido ao hiperparatireoidismo secundário, podendo mimetizar a FSN. A biópsia do tecido lesado tem papel fundamental no diagnóstico. Entretanto, já foram descritos casos de FSN associados à calcifilaxia ${ }^{30,31}$.

Mixedema pré-tibial - a ausência de placas ou nódulos arroxeados, principalmente em membros inferiores, associados a hipertireoidismo (Doença de Graves) na FSN também descartam esta patologia28.

\section{Tratamento}

Até o momento, não existe nenhuma terapêutica comprovada para a FSN ${ }^{16,32}$. A melhora da função renal em pacientes com disfunção renal aguda parece ter efeito de diminuir a progressão e até mesmo parar e reverter o processo de fibrose. ${ }^{6,28,29,32-35}$ Dessa forma, o emprego de estratégias com intuito de melhorar a função renal, no caso de insuficiência renal aguda, e tratar a doença de base, deve ser estimulado. Além disso, o emprego de fisioterapia em todos os pacientes acometidos deve ser estimulado com intuito de diminuir as contraturas patológicas e a dor crônica ${ }^{17}$.

Entretanto, existem diversos relatos na literatura de tentativas terapêuticas sistêmicas e tópicas que obtiveram aparente sucesso em casos isolados.

Diversos relatos apontam a recuperação clínica após o transplante renal bem-sucedido, tanto com doadores falecidos como com doadores vivos ${ }^{6,17,36}$, provavelmente devido a uma melhora da função renal e a excreção do gadolínio, conforme citado anteriormente.

O corticosteróide, tanto sistêmico quanto tópico, foi empregado sem que se demonstrasse melhora nas lesões ou dos sintomas associados, como dor crônica ${ }^{2,37}$.

Baron et al. ${ }^{34}$ reportaram melhora clínica após plasmaférese, não constatada por Hubbard et al. ${ }^{38}$.

A talidomida foi empregada em alguns casos isolados com resultados díspares ${ }^{11,36}$.

Devido ao benefício já comprovado em outras desordens fibrosantes, a pentoxifilina foi testada com resposta clínica favorável por Chung ${ }^{39}$. Mais casos semelhantes serão necessários para comprovação de seu real benefício. A ciclofosfamida não apresentou benefício nos relatos da literatura ${ }^{16,35,38}$.

Devido às suas propriedades quelantes e antioxidantes, 0 tiossulfato de sódio foi testado recentemente por Yerram et al. ${ }^{31}$, com resultado satisfatório em uma paciente que recebeu 12,5 gramas, três vezes por semana, por três meses.

Chung ${ }^{39}$ relatou um caso com resposta favorável com o uso da imunoglobulina; entretanto, não descreveu se houve concomitante melhora da função renal.

Com terapêutica tópica, foram empregados diversos tipos de fototerapia, como ultravioleta, fotoferese e terapia fotodinâmica, com resultados promissores. 0 mecanismo de ação permanece incerto, mas aparentemente há diminuição da síntese de colágeno por supressão de citocinas próinflamatórias 6,40-44.

\section{Prevenção e recomendações finais}

A FSN é uma doença devastadora, que acomete a pele e diversos tecidos do corpo. Embora não se tenha certeza sobre sua fisiopatogênese, os dados existentes na literatura permitem sugerir recomendações visando prevenção ou mesmo minimizar os riscos para se adquirir esta doença.

0 primeiro e mais correto é sugerir que todo paciente a ser submetido a ressonância magnética deva ser avaliado quanto à presença de insuficiência renal e com isso identificar os que possuem um risco aumentado de desenvolver FSN, ou seja: ${ }^{45,46}$

- pacientes com insuficiência renal aguda, principalmente associado à insuficiência hepática;

- pacientes transplantados renais ou hepáticos e com doença renal;

- pacientes com doença renal estágio 4 ou 5 e sem terapia dialítica; ${ }^{21,27,32}$

- pacientes em hemodiálise ou diálise peritoneal.

Para aqueles pacientes com creatinina sérica elevada é recomendável que se estime a função renal, visando a segurança na realização do exame de ressonância magnética.

Identificado o grupo de risco de desenvolver FSN, preconizase evitar o uso do gadolínio a não ser que este exame seja imprescindível ${ }^{21,27,32,46,47}$

Se o exame de ressonância magnética com gadolínio for realmente essencial, recomenda-se o uso da menor dose possível, 11,27,32,46,47 evitando os quelantes de gadolínio lineares e múltiplas exposições ao gadolínio. 0 paciente deve ser informado do risco, benefício e alternativas. Todo paciente de risco que for exposto a exame contrastado deve ter uma avaliação clínica evolutiva. Deve-se evitar o uso do gadolínio em pacientes com insuficiência renal aguda até a recuperação da função renal ${ }^{46,47}$.

Entre os pacientes com doença renal crônica em hemodiálise e que foram expostos ao gadolínio, recomenda-se realizar três sessões de hemodiálise, iniciando a primeira sessão até três horas após a exposição. Deve-se enfatizar que a eficácia da hemodiálise em prevenir a FSN é desconhecida ${ }^{46}$.

Aos pacientes portadores de doença renal crônica em diálise peritoneal expostos ao gadolínio, recomenda-se aumentar o número de trocas de bolsas de diálise ou aumentar a prescrição da diálise peritoneal automática por pelo menos 48 horas e considerar realização de hemodiálise naqueles que possuírem um acesso vascular, forem expostos a altas ou múltiplas doses do gadolínio ou caso o nefrologista julgue necessário ${ }^{46}$.

Já entre os pacientes com insuficiência renal aguda ou crônica estágio 4 ou 5 e que não estão em diálise e foram expostos ao gadolínio, até o momento não há como estimar 0 risco de desenvolver FSN, sendo que a decisão de realizar hemodiálise deve ser individualizada avaliando os riscos e benefícios numa decisão conjunta entre o nefrologista, o paciente e o seu clínico46.

Concluindo, a FSN é uma doença progressiva e potencialmente fatal descrita em pacientes com insuficiência renal aguda ou crônica, com filtração glomerular $<30 \mathrm{~mL} / \mathrm{min} / 1,73 \mathrm{~m}^{2}$ e expostos a contrastes contendo gadolínio. Até o momento, não há uma terapia ou combinação de terapias que mostre um benefício consistente na FSN quanto à recuperação da função 
renal, a não ser em pacientes com insuficiência renal aguda com recuperação da função renal. Embora não provado, pacientes submetidos a transplante renal parecem apresentar uma melhora do quadro da FSN.

\section{Conflito de interesse: não há}

\section{SUMMARY}

\section{Nephrogenic systemic fibrosis: a seVere complication of USE to GADOLINIUM IN PATIENTS WITH KIDNEY FAILURE}

Nephrogenic systemic fibrosis (NSF), also known as nephrogenic fibrosing dermopathy (NFD), is a condition that has occurred only in patients with renal insufficiency. Besides lesions of the skin, this syndrome include fibrosis of skeletal muscle, joints, liver, lung, and heart, with possible fatal outcomes. This disease was first described in 1997 and several reports described the development of NSF after the exposure to gadolinium-based magnetic resonance imaging contrast agents. This review aims to alert physicians and nephrologists about this new pathology that affects patients with renal dysfunction, describing its demographic and epidemiologics aspects, clinic presentation, diagnosis and prognosis, beyond options to prevent and current treatment. We concluded that in all patient with elevated serum creatinine physicians should estimade his kidney function (creatinine clearence) in order to safety of magnetic resonance. [Rev Assoc Med Bras 2009; 55(2): 220-5]

KEY WORDS: Nephrogenic systemic fibrosis. Nephrogenic fibrosing dermopathy. Gadolinium. Fibrosis. Kidney failure.

\section{ReferênCIAS}

1. Cowper SE, Robin HS, Steinberg SM, Su LD, Gupta S, LeBoit PE. Scleromyxedema-like cutaneous disease in renal-dialysis patients. Lancet. 2000;356:1000-1.

2. Ting WW, Stone MS, Madison KC, Kurtz K. Nephrogenic fibrosing dermopathy with systemic involvement. Arch Dermatol. 2003;139:903-6.

3. Jiménez SA, Artlett CM, Sandorfi N, Derk C, Latinis K, Sawaya $H$, et al. Dialysis-associated systemic fibrosis (nephrogenic fibrosing dermopathy): study of inflammatory cells and transforming growth factor beta 1 expression in affected skin. Arthritis Rheum. 2004;50:2660-6.

4. Kucher C, Steere J, Elenitsas R, Siegel DL, Xu X. Nephrogenic fibrosing dermopathy/nephrogenic systemic fibrosis with diaphragmatic involvement in a patient with respiratory failure. J Am Acad Dermatol. 2006;54:31-4.

5. Cowper SE. Nephrogenic fibrosing dermopathy [NFD/NSF website]. 20012007. [cited 2007 J un 2009]. Available from: http://www.icnfdr.org

6. Mackay-Wiggan JM, Cohen DJ, Hardy MA, Knobler EH, Grossman ME. Nephrogenic fibrosing dermopathy (scleromyxedema-like illness of renal disease). J Am Acad Dermatol. 2003;48:55-60.

7. McNeill AM, Barr RJ. Scleromyxedema-like fibromucinosis in a patient undergoing hemodialysis. Int J Dermatol. 2002;41:364-7.

8. Sadowski EA, Bennett LK, Chan MR, Wentland AL, Garrett AL, Garrett RW, et al. Nephrogenic systemic fibrosis: risk factors and incidence estimation. Radiology. 2007;243:148-57

9. The International Center for Nephrogenic fibrosing dermopathy research. [cited 2007 march]. Available from: http://www.icnfdr.org.

10. Auron A, Shao L, Warady BA. Nephrogenic fibrosing dermopathy in children. Pediatr Nephrol 2006;21:1307-11.

11. Moschella SL, Kay J, Mackool BT, Liu V. Case records of the Massachusetts General Hospital. Weekly clinicopathological exercises. Case 35-2004. A 68-year-old man with end stage renal disease and thickening of the skin. $\mathrm{N}$ Engl J Med. 2004;351:2219-27.
12. Daram SR, Cortese CM, Bastani B. Nephrogenic fibrosing dermopathy/ nephrogenic systemic fibrosis: report of a new case with literature review. Am J Kidney Dis. 2005;46:754-9.

13. Cowper SE. Nephrogenic fibrosing dermopathy: the first 6 years. Curr Opin Rheumatol. 2003;15:785-90.

14. Cowper SE, Bucala R, LeBoit PE. Case 35-2004: nephrogenic fibrosing dermopathy. N Engl J Med. 2005;352:1723-4

15. Centers for Disease Control and Prevention (CDC). Neprogenic fibrosing dermopathy associated with exposure to gadolinium-containing contrast agents - St. Louis, Missouri, 2002-2006. MMWR Morb Mortal Wkly Rep. 2007;56:137-41.

16. Mendoza FA, Artlett CM, Sandorfi N, Latinis K, Piera-Velazquez S, J imenez SA. Description of 12 cases of nephrogenic fibrosing dermopathy and review of the literature. Semin Arthritis Rheum. 2006:35:238-49.

17. Grobner T. Gadolinium-a specific trigger for the development of nephrogenic fibrosing dermopathy and nephrogenic systemic fibrosis? Nephrol Dial Transplant. 2006;21:1104-8.

18. Marckmann P, Skov L, Rossen K, Dupont A, Damholt MB, Heaf J G, et al. Nephrogenic systemic fibrosis: suspected causative role of gadodiamide used for contrast-enhanced magnetic resonance imaging. J Am Soc Nephrol. 2006;17:2359-62.

19. Stenver DI. Investigation of the safety of MRI contrast medium Omniscan. Danish Medicines Agency 2006 May 29. [cited jul 12]. Available from: http:/ /www.dkma.dk/visUKLSArtikel. asp?artikelID $=8931$.

20. Cowper SE, Bucala R. Nephrogenic fibrosing dermopathy: suspect identified, motive unclear. Am J Dermatopathol. 2003;25:358.

21. Perazella MA, Rodby RA. Gadolinium use in patients with kidney disease: $A$ cause for concern. Semin Dial. 2007;20:179-85.

22. Runge VM. Safety of magnetic resonance contrast media. Top Magn Reson Imaging. 2001;12:309-14.

23. J offe P, Thomsen HS, Meusel M. Pharmacokinetics of gadodiamide injection in patients with severe renal insufficiency and patients undergoing hemodialysis or continuous ambulatory peritoneal dialysis. Acta Radiol. 1998:5:491-502.

24. LeBoit PE. What nephrogenic fibrosing dermopathy might be. Arch Dermatol. 2003;139:928-30.

25. Swaminathan S, Ahmed I, McCarthy JT, Albright RC, Pittelkow MR, Caplice $\mathrm{NM}$, et al. Nephrogenic fibrosing dermopathy and high-dose erythropoietin therapy. Ann Intern Med. 2006;145:234-5.

26. Galan A, Cowper SE, Bucala R. Nephrogenic systemic fibrosis (nephrogenic fibrosing dermopathy). Curr Opin Rheumatol. 2006;18:614-7.

27. Deo A, Fogel M, Cowper SE. Nephrogenic systemic fibrosis: A population study examining the relationship of disease development to gadolinium exposure. Clin I Am Soc Nephrol. 2007:2:264-7.

28. Swartz RD, Crofford LJ, Phan SH, Ike RW, Su LD. Nephrogenic fibrosing dermopathy: a novel cutaneous fibrosing disorder in patients with renal failure. Am J Med. 2003;114:563-72.

29. Jan F, Segal J M, Dyer J, LeBoit P, Siegfried E, Frieden IJ. Nephrogenic Fibrosing Dermopathy: Two Pediatric Cases. J Pediatr. 2003;143:678-81.

30. Edsall LC, English J C 3rd, Teaque MW, Patterson JW. Calciphylaxis and metastatic calcification associated with nephrogenic fibrosing dermopathy. J Cutan Pathol. 2004;31:247-53.

31. Yerram P, Saab G, Karuparthi PR, Hayden MR, Khanna R. Nephrogenic systemic fibrosis: A mysterious disease in patients with renal failure: role of gadolinium-based contrast media in causation and the beneficial effect of intravenous sodium thiosulfate. Clin J Am Soc Nephrol. 2007;2:258-63.

32. Food and Drug Administration [Cited 2007 jul 12] Available from: http:// www.fda.gov/cder/drug/advisory/gadolinium.

33. Cowper SE, Su L, Robin H, Bhawan J, LeBoit PE. Nephrogenic fibrosing dermopathy. Am J Dermatopathol. 2001;23:383-93.

34. Baron PW, Cantos K, Hillebrand DJ , Hu KQ, Ojogho ON, Nehlsen-Cannarella $\mathrm{S}$, et al. Nephrogenic fibrosing dermopathy after liver transplantation successfully treated with plasmapheresis. Am J Dermatopathol. 2003;25:204-9

35. Tan AW, Tan SH, Lian TY, Ng SK. A case of nephrogenic fibrosing dermopathy. Ann Acad Med Singapore. 2004;33:527-29

36. Streams BN, Liu V, Liégeois N, Moschella SM. Clinical and pathologic features of nephrogenic fibrosing dermopathy: a report of two cases. J Am Acad Dermatol. 2003;48:42-7.

37. Hancox J G, Mengesha YM, Sanqueza OP, Yosipovitch G. Nephrogenic fibrosing dermopathy after five days of hemodialysis. J Drugs Dermatol. 2003;2:550-3. 
38. Hubbard V, Davenport A, J armulowicz M, Rustin M. Scleromyxoedema-like changes in four renal dialysis patients. Br J Dermatol. 2003;148:563-8.

39. Chung $\mathrm{HJ}$, Chung KY. Nephrogenic fibrosing dermopathy: response to highdose intravenous immunoglobulin. BrJ Dermatol. 2004;150:596-7.

40. Gilliet M, Cozzio A, Burg G, Nestle FO. Successful treatment of three cases of nephrogenic fibrosing dermopathy with extracorporeal photopheresis. $\mathrm{Br}$ J Dermatol. 2005;152:531-6.

41. Lauchli S, Zortea-Caflisch C, Nestle FO, Burg G, Kempf W. Nephrogenic fibrosing dermopathy treated with extracorporeal photopheresis. Dermatology. 2004;208:278-80.

42. Kafi R, Fisher GJ, Quan T, Shao Y, Wang R, Voorhees JJ, et al. UV-A1 phototherapy improves nephrogenic fibrosing dermopathy. Arch Dermatol. 2004;140:1322-4.

43. Schmook T, Budde K, Ulrich C, Neumayer HH, Fritsche L, Stockfleth E. Successful treatment of nephrogenic fibrosing dermopathy in a kidney transplant recipient with photodynamic therapy. Nephrol Dial Transplant. $2005 ; 20: 220-2$
44. Wahba IM, White K, Meyer M, Simpson EL. The case for ultraviolet light therapy in nephrogenic fibrosing dermopathy- report of two cases and review of the literature. Nephrol Dial Transplant. 2007;22:631-6.

45. Perazella MA. Nephrogenic systemic fibrosis, kidney disease, and gadolinium: is there a link? Clin J Am Soc Nephrol. 2007;2:200-2.

46. Perazella MA, Reilly RF. Nephrogenic systemic fibrosis: recommendations for gadolinium-based contrast use in patients with kidney disease. Semin Dial. 2008;21:171-3.

47. Perazella MA. How should nephrologists approach gadolinium-based contrast imaging in patients with kidney disease? Clin J Am Soc Nephrol. 2008;3:649-51.

Artigo recebido: 29/10/07

Aceito para publicação: 29/06/08 